Relations industrielles

Industrial Relations

\title{
Les travailleurs du Québec au XXe siècle
}

\section{Léo Roback}

Volume 40, numéro 4, 1985

URI : https://id.erudit.org/iderudit/051380ar

DOI : https://doi.org/10.7202/051380ar

Aller au sommaire du numéro

Éditeur(s)

Département des relations industrielles de l'Université Laval

ISSN

0034-379X (imprimé)

1703-8138 (numérique)

Découvrir la revue

Citer cet article

Roback, L. (1985). Les travailleurs du Québec au XXe siècle. Relations industrielles / Industrial Relations, 40(4), 826-846.

https://doi.org/10.7202/051380ar

Tous droits réservés (C Département des relations industrielles de l'Université Laval, 1985
Ce document est protégé par la loi sur le droit d'auteur. L'utilisation des services d'Érudit (y compris la reproduction) est assujettie à sa politique d'utilisation que vous pouvez consulter en ligne.

https://apropos.erudit.org/fr/usagers/politique-dutilisation/ 


\title{
Les travailleurs du Québec au XXe siècle
}

\author{
Léo Roback
}

Léo Roback est décédé à Montréal le 27 juin 1985 . Il avait écrit le texte suivant, en anglais, pour un symposium, tenu à l'Université Memorial, à Saint-Jean, TerreNeuve, le 9 février 1983. L'ensemble des travaux du symposium vient d'être publié "Ả la mémoire de Léo Roback», sous le titre de Lectures in Canadian Labour and Working-Class History, par le Comité d'histoire des travailleurs canadiens. Nous publions cette traduction (inédite) du dernier texte de Léo Roback dans un double but: rendre le document accessible aux lecteurs francophones et rendre, en même temps, un hommage posthume à son auteur pour son importante contribution à l'histoire et à la pratique du syndicalisme au Québec. La traduction est du professeur Gérard Hébert, de l'École des relations industrielles de l'Université de Montréal.

L'auteur résume les grandes étapes du mouvement ouvrier au Québec depuis le début du siècle en les situant dans le contexte économique, social et politique de chaque période, et en soulignant quelques-uns de ses thèmes préférés: les diverses strates dans la classe ouvrière elle-même, l'insuffisance chronique du revenu moyen des salariés pour leur assurer une vie décente, l'opposition systématique des employeurs et des gouvernements au mouvement syndical.

Le Québec est entré dans le vingtième siècle avec fracas. La longue dépression des années 1880 et 1890 a fait place à une période presque ininterrompue d'expansion économique et d'inflation jusqu'à la dépression de l'après-guerre.

L'industrialisation au Québec a pris son essor pendant la première décennie du siècle nouveau. A la structure industrielle existante, constituée principalement des textiles, du vêtement, de la chaussure, du tabac, des fabriques de beurre et de fromage, des moulins à scie et des moulins à grain, vinrent s'ajouter, dans une croissance rapide, les pouvoirs hydro-électriques et de nouvelles industries, qui profitèrent largement de l'électricité à bon marché et des ressources naturelles; ce fut le cas des industries de la pulpe et du papier, de la chimie lourde et de la métallurgie.

Parallèlement, on a vu croître le nombre des adhésions au mouvement syndical et l'activité de grève. Les deux phénomènes ont atteint des records historiques en 1919.

- ROBACK, Léo (1918-1985), Ecole des relations industrielles, Université de Montréal. 
Une des caractéristiques de la période fut la mobilité démographique. Les surplus de population des régions rurales du Québec - accrus par le développement de l'industrie laitière, qui requiert moins de main-d'oeuvre que l'agriculture traditionnelle - accouraient vers les villes de Montréal et de Québec, surtout vers Montréal: Montréal croissait alors de plus en plus vite. D'un autre côté, le développement industriel du Québec et de l'Ontario, ainsi que la poussée du blé dans l'Ouest canadien, attiraient des centaines de milliers d'immigrants du Royaume-Uni et de tous les coins de l'Europe. La plupart arrivaient à Montréal et plusieurs, bien que ce ne fut pas la majorité, y demeuraient. Ils s'ajoutaient aux vagues précédentes d'immigration, particulièrement à celle des Irlandais, qui avaient quitté leur patrie par suite de la «famine de patates» au milieu du XIX ${ }^{\mathrm{e}}$ siècle. Les immigrants irlandais constituèrent le premier noyau du prolétariat urbain de Montréal; ils fournissaient la main-d'oeuvre non spécialisée que requéraient le port, la construction des chemins de fer et des canaux. Ils formaient la majorité des 20 travailleurs tués par les troupes britanniques, le 11 juin 1843, à Saint-Timothée, près de Valleyfield, durant la grève des ouvriers de la construction du canal de Beauharnois.

Au début du $\mathrm{XX}^{\mathrm{e}}$ siècle, la classe ouvrière des grands centres urbains du Québec provenait de quatre sources principales. Dans leur excellente Histoire du Québec contemporain, Linteau, Durocher et Robert identifient les quatre catégories suivantes, qui correspondent à ces quatre sources:

1. Les anciens travailleurs autonomes, ou artisans, ruinés et supplantés par l'arrivée de la production capitaliste

2. Les ouvriers non qualifiés déjà dans les grandes villes. Les nombreux journaliers (hommes) et domestiques (femmes) constituaient des recrues toute désignées pour le travail dans les manufactures. tures.

3. Les immigrants d'Irlande, du Royaume-Uni et d'Europe. Plusieurs d'entre eux étaient d'anciens artisans ou d'anciens ouvriers qualifiés, beaucoup provenant du milieu rural ou semi-rural.

4. Les immigrants internes, qui venaient des fermes et des villages du Québec. Habitués à des conditions de vie tout près du niveau de subsistance, sans connaissances ni habiletés techniques, trop nombreux pour avoir quelque pouvoir de négociation que ce soit, ils fournissaient un réservoir tout prêt de travail non qualifié ou non spécialisé.

\section{Les strates dans la classe ouvrière}

Il y avait une sorte de stratification à l'intérieur même de la classe ouvrière, urbaine et industrielle. Au sommet de la pyramide, on trouvait les aristocrates du travail: les ouvriers qualifiés de la construction et de la métallurgie, les travailleurs des métiers de l'imprimerie, les employés itinérants des chemins de fer, les mécaniciens responsables de la machinerie dans les différentes fabriques. Ils bénéficiaient d'un niveau de salaire et d'une permanence d'emploi bien supérieurs à la grande majorité des travailleurs. Ils formaient aussi le noyau des premiers membres et des premiers dirigeants du mouvement syndical. Avec eux, on retrouvait les employés de bureau qui occupaient les positions supérieures, tant dans l'entreprise privée que dans l'administration publique. 
Une seconde strate, juste au-dessous, était constituée des travailleurs à la production semi-qualifiés. C'est dans ce groupe qu'on retrouvait le plus grand nombre de femmes qui travaillaient en usine. Exclus des syndicats de métier déjà bien établis, ces travailleurs devaient se contenter de faibles salaires, faire de longues heures, subir de fréquentes périodes de chômage, saisonnier et cyclique, et connaître le risque des accidents et des maladies professionnelles.

Au bas de l'échelle se trouvait la masse des ouvriers non qualifiés. À peine lettrés, parfois complètement illettrés, ils travaillaient généralement de façon occasionnelle, au taux de salaire que chaque employeur voulait bien leur offrir. On les retrouvait comme journaliers, soit sur les chantiers de construction, soit dans les ports, le transport ou les services municipaux. Ã quelques exceptions près, comme les débardeurs, ils n'avaient que peu de liens avec les syndicats ou les unions.

Les travailleurs canadiens-français, qui constituaient les trois-quarts de la main-d'oeuvre, se trouvaient concentrés dans les deux catégories inférieures. Le groupe supérieur - composé des hommes de métiers spécialisés, des surintendants et de tous ceux qui avaient des postes de responsabilité dans la direction et les bureaux - était formé, dans une proportion bien supérieure à leur nombre, de ceux que le recensement appelait les personnes d'origine britannique. Leur situation tenait, entre autres, au fait que l'anglais constituait la langue de travail dans toutes les grandes entreprises, même dans toutes les usines et les bureaux où le patron était de langue anglaise. Le contrôle quasi total de l'emploi dans le secteur privé par des industriels et des financiers de langue anglaise était alors considéré comme une donnée avec laquelle il fallait compter. Le probleme de la langue soulevait les passions même dans le monde syndical, particulièrement en dehors de Montréal, où les unions internationales essayaient d'organiser une main-d'oeuvre presque entièrement francophone. Pendant plusieurs décennies, jusqu'à la fin des années 1960 , plusieurs milliers de syndiqués francophones durent accepter le fait que leurs représentants syndicaux ne leur parlent qu'anglais, négocient pour eux en anglais, avec des patrons anglophones, et leur transmettent des conventions collectives exclusivement anglaises. Pour ajouter l'insulte à l'injure, on les congédiait également en anglais.

Les travailleurs canadiens-français étaient sous-représentés dans les classes supérieures du monde ouvrier, et le sous-développement du système public d'éducation, par comparaison avec celui dont jouissait la majorité anglophone, ne pouvait que perpétuer une telle situation. Même dans les années 1950, le premier ministre Duplessis rappelait aux commissions scolaires catholiques que leur mandat se limitait aux écoles élémentaires. Une étude sur les décrocheurs dans les deux systèmes scolaires de Montréal, au cours de la première Grande Guerre, a montré que, dans les écoles catholiques, généralement de langue française, $75 \%$ des enfants qui avaient commencé l'école l'avaient quittée en quatrième année; par contre, dans les écoles anglo-protestantes, le taux de départ au niveau de la quatrième année n'était que de $25 \%$. Terry Copp a montré que la majorité de l'élite canadienne-française n'était pas intéressée plus qu'il fallait à un système public d'éducation, parce que leurs enfants à eux fréquentaient les collèges 
classiques; ces collèges étaient des écoles privées, sur le modèle des lycées français; ils ont constitué, pendant plusieurs décennies, le seul chemin qui menait à l'université. En contraste, la majorité des enfants anglophones de toutes les classes, y compris les plus aisées, fréquentaient le système d'éducation public protestant.

Les milliers d'enfants au travail, environ $6 \%$ de toute la main-d'oeuvre à Montréal, au tournant du siècle, fournissaient un réservoir supplémentaire, particulièrement peu exigeant quant au salaire et soumis à une discipline très rude. La législation qui limitait le travail des enfants n'était tout simplement pas appliquée; en fait, elle ne pouvait pas l'être. Les parents avaient besoin de ce revenu supplémentaire, et les employeurs recherchaient le travail des petites mains peu dispendieuses. En 1907, la loi fut modifiée pour interdire l'embauche de tout enfant au-dessous de 14 ans et pour obliger les enfants illettrés de moins de 16 ans à fréquenter l'école du soir. L'année suivante, l'inspecteur Guyon ${ }^{1}$ fit rapport qu'il était difficile d'appliquer la nouvelle disposition, puisqu'en plusieurs endroits il n'y avait pas d'école du soir, et que là où il y en avait, elles ne recevaient que des garçons. Les enfants dans les manufactures travaillaient le même nombre d'heures que les adultes. Dans les fabriques de coton, cela voulait dire 11 heures par jour et 5 heures le samedi, à un salaire de $0.061 / 2$ de l'heure. Montréal était le pôle d'attraction de l'industrie et de la finance au Canada, encore plus au Québec. Le port était à la fois un facteur de stimulation et de restriction. La fermeture du port pendant les quatre mois d'hiver signifiait qu'une bonne partie de l'activité économique de Montréal et de Québec se limitait à huit mois de l'année. Le chômage saisonnier et les coupures de salaire faisaient partie de la vie tout autant que les tempêtes de neige.

\section{L'industrialisation en province}

Le départ véritable de l'industrialisation, au cours des deux premières décennies du siècle, fit surgir de grands établissements en dehors de Montréal. Des moulins de pulpe et de papier jaillirent dans la vallée du Saint-Maurice et dans la région du Saguenay-Lac Saint-Jean. Des chantiers maritimes et des usines de fabrication pour les chemins de fer s'établirent et se développèrent rapidement, pour répondre aux besoins créés par la poussée du blé dans l'Ouest et par la guerre. Les développements technologiques nouveaux amenèrent des usines d'aluminium à Shawinigan Falls et diverses fabriques d'équipements électriques en d'autres endroits; on y produisait en particulier les moteurs dont l'usage se répandait partout, et ce nouvel appareil-miracle qui s'appelait le téléphone.

Le développement économique qui marqua les années juste avant la guerre et pendant la guerre a créé quelques millionnaires et certains empires industriels et financiers. Les travailleurs du Québec ne participèrent pas aux bénéfices qui en découlèrent. Au contraire, les documents disponibles montrent plutôt que la majorité des familles de la classe ouvrière pouvaient à peine joindre les deux bouts. Ceci résultait principalement des salaires, qui

1 Louis Guyon fut le premier inspecteur du travail au Québec. Nommé en 1888, il le demeura jusqu'à la fin des années 1920. (Note du traducteur.) 
ne dépassaient guère le niveau de subsistance, et des fréquentes périodes de chômage. Les récessions, qui revenaient de façon cyclique, comme celle des années 1913 à 1915, aggravèrent la situation à un moment où le cout de la vie grimpait sérieusement, annonçant en quelque sorte la terrible inflation des dernières années de la guerre et de l'immédiat après-guerre.

En 1900, on estimait qu'une famille ouvrière de cinq personnes avait besoin, à Montréal, d'un minimum de $9.64 \$$ pour se procurer les choses essentielles de la vie, la nourriture, le toit et le vêtement; pour répondre à l'ensemble de ses besoins, il fallait à une telle famille 13.77\$. Cette année-là, les revenus annuels moyens des ouvriers, en excluant les plus pauvres, s'élevaient à 7.78\$. En 1911, toujours selon le ministère du Travail, le budget familial requis était, pour les nécessités de la vie, de $12.82 \$$ et, pour l'ensemble des besoins de la famille, de 18.31\$; les revenus hebdomadaires moyens des chefs de famille n'atteignaient que $10.21 \$$ pour un journalier de la construction, et 13.70\$ pour un homme de métier dans le bâtiment. Seuls les serre-freins des chemins de fer dépassaient la ligne de pauvreté avec 18.67\$. De 1900 à 1913, le coût de la vie monta d'environ 43\%, de 1915 à 1920 , il bondit de $80 \%$.

\section{Les conflits ouvriers}

Le contexte économique et les conditions sociales des travailleurs étaient favorables à la syndicalisation et au militantisme. Il s'y ajoutait un facteur décisif, le regroupement des unions dans la Fédération américaine du travail (F.A.T.); en effet, on venait d'assister à l'élimination du groupe rival de la F.A.T., les Chevaliers du travail, d'abord aux Etats-Unis, puis au Québec, dernier bastion des chevaliers. Les années d'avant la guerre furent très actives: il y eut beaucoup d'organisation syndicale et plusieurs grèves, particulièrement dans le bâtiment et les chemins de fer, mais aussi dans des secteurs de production de masse comme les textiles de coton, le vêtement et la chaussure, les scieries, ainsi que chez les débardeurs du port de Montréal. Le contexte d'alors - les employeurs avaient la main libre pour bloquer ou détruire les unions, avec l'appui de la police et de l'appareil judiciaire - ne donnait aux syndicats du temps qu'un espoir de vie relativement court. Malgré tout, le nombre des succursales locales d'unions au Québec a doublé entre 1901 et 1911; le nombre de membres dépassait 15000 en 1911, probablement trois ou quatre fois celui de 1901. Durant l'année turbulente de 1919 , on a dénombré plus de 500 sections locales et plus de 60000 membres.

Le type de grèves de cette période reflétait les conditions économiques. Les grèves offensives, pour obtenir des augmentations de salaire ou pour faire reconnaître l'union, ou encore pour réduire le nombre d'heures de travail, ont caractérisé les années de prospérité de 1901, 1903, 1907, 1910 et 1912-1913. Les conflits de nature défensive, contre les réductions de salaire ou le prolongement des heures, correspondent aux années de récession, comme 1902 et 1908.

Deux conflits majeurs impliquèrent l'Association internationale des Machinistes et les chemins de fer. En 1905, la grève contre le Grand Trunk Railway dura 9 mois et fut perdue. En 1908, 8000 syndiqués, machinistes et 
wagonniers, firent la grève contre le Canadien Pacifique pour empêcher les réductions de salaire et conserver aux comités ouvriers le droit de rencontrer la direction. De nouveau, les briseurs de grève firent échouer l'arrêt de travail.

En 1903, plus de 2000 débardeurs du port de Montréal menèrent une grève de six semaines, qui tourna en une petite guerre civile. Des soldats de l'armée régulière vinrent de Toronto relever leurs collègues de Montréal. Les compagnies de navigation amenèrent un millier de briseurs de grève du Royaume-Uni pour décharger les navires. Â la fin, l'union réussit à obtenir sa reconnaissance et une augmentation de $10 \%$; ce fut grâce à la solidarité des débardeurs des principaux ports de la côte est du Canada et des EtatsUnis.

Une grève amère et sanglante, dans un moulin à scie de Buckingham, à l'automne de 1906, impliqua 400 ouvriers de l'union des charpentiers. Des gardes de sécurité privés tirèrent sur les grévistes; ils en tuèrent deux et en blessèrent plusieurs autres; un agent fut lui-même tué. La milice fut dépêchée d'Ottawa (située juste à 50 milles à l'ouest) et l'armée régulière, de Saint-Jean, Québec. La grève fut perdue.

L'industrie du textile de coton fit l'objet d'au moins 40 grèves et lockout entre 1900 et 1908 . Quelques-unes avaient pour objet d'obtenir la reconnaissance de l'union, affiliée à la Fédération américaine du travail; celle-ci enregistra d'importants succès partout au Québec, en 1906; mais elle disparut aussi rapidement quand 14 succursales locales en quittèrent les rangs, pour former une organisation syndicale canadienne.

\section{Les syndicats catholiques}

Le conflit dans l'industrie de la chaussure à Québec, en 1900 et 1901, a une importance toute particulière, parce qu'il marquait le début d'un mouvement qui allait devenir la Confédération des Travailleurs catholiques du Canada, aujourd'hui la Confédération des Syndicats nationaux (CSN). Le conflit prit la forme d'un lockout et il toucha presque toute l'industrie. Une vingtaine d'employeurs, la plupart canadiens-français, fermèrent leurs usines, parce qu'ils ne voulaient plus traiter avec l'union existante, autrefois affiliée aux Chevaliers du travail. Le travail reprit grâce à l'intervention de l'archevêque Monseigneur (plus tard le Cardinal) Bégin, qui fit office d'arbitre. Monseigneur Bégin décida que le droit des travailleurs à se regrouper en syndicats était un droit naturel, comme l'avait proclamé Léon XIII dans son encyclique Rerum Novarum en 1891. Toutefois, ce droit des travailleurs exigeait que ceux-ci acceptent de ne poursuivre que de justes objectifs; ils devaient ainsi reconnaître qu'il existait une harmonie naturelle entre les intérêts de l'employeur et ceux des travailleurs, et rejeter la lutte des classes. L'union dut adopter une nouvelle constitution, qui incorporait ces éléments de la doctrine sociale de l'Église, et accepter la présence d'un aumônier; celui-ci jouissait d'une autorité considérable sur tout ce qui touchait à la doctrine et à la morale, comme sur la question des grèves auxquelles il ne fallait recourir qu'en dernier ressort. 
Ce fut là le début modeste et fragile du syndicalisme catholique au Québec. Il se développa lentement et difficilement jusqu'à la fin de la seconde Guerre mondiale. Les divers syndicats se regroupèrent en 1921 pour former la Confédération des travailleurs catholiques du Canada (C.T.C.C.), et les pionniers du mouvement auraient de la difficulté à reconnaître dans la CSN d'aujourd'hui leur propre rejeton.

Dans cette première période, le mouvement syndical catholique était, d'abord et avant tout, un mouvement idéologique. Il s'était donné pour mission de convaincre les travailleurs et les employeurs catholiques du bienfondé de la doctrine sociale de l'Église, présentée par le pape Léon XIII, et de travailler à son application. La province de Québec paraissait un terrain particulièrement favorable à cette tentative, parce que la population y était relativement homogène et que l'autorité morale de l'Église y était grande. Rapidement, cependant, les théoriciens et les praticiens du mouvement rencontrèrent de sérieux problèmes. Les relations du travail au Québec ressemblaient à celles du reste de l'Amérique du Nord. La majorité des syndiqués canadiens-français appartenait aux unions internationales, qu'on qualifiait de neutres, socialistes et étrangères. D'un autre côté, la plupart des employeurs canadiens-français étaient convaincus, comme tous leurs collègues, que le seul bon syndicat est un syndicat mort. De plus, l'homogénéité religieuse était loin d'être complète, puisque les travailleurs catholiques anglophones se tournaient plutôt vers les internationales. En fait, les syndicats catholiques trouvèrent leur véritable fondement dans l'identité ethno-linguistique du groupe canadien-français, ce dont témoigne l'usage de plus en plus répandu de l'expression "syndicats nationaux» pour en désigner les diverses unités.

En pratique, les syndicats catholiques reçurent un appui considérable de la part de l'Église. À Montréal, le château-fort des unions internationales, les entrepreneurs et les fournisseurs des églises, des écoles, des hôpitaux et des autres institutions rattachées à l'Église étaient fortement invités à accorder aux membres des syndicats catholiques une forme d'atelier fermé. On trouvait quelque chose de semblable dans l'imprimerie.

\section{Les aspects politiques}

Les deux premières décennies du siècle ont connu deux autres développements majeurs de grande importance. Ce fut d'abord la fondation, dès le début du siècle, du parti ouvrier de Québec. Le mouvement prit naissance dans les divers Conseils des métiers et du travail de la région de Montréal.

Un réseau de cercles ouvriers dans les différents quartiers populaires de la ville s'avéra un des éléments les plus dynamiques. Le programme du parti en était un de réforme sociale. On mettait l'emphase particulièrement sur la législation qui devait protéger les travailleurs contre l'exploitation et leur assurer de meilleures conditions de travail et de vie. L'éducation obligatoire et gratuite, un réseau de bibliothèques publiques, le bannissement de tout travail des enfants, l'indemnisation des victimes d'accidents du travail, les pensions de vieillesse et l'assurance-maladie constituaient autant d'objectifs dans le programme du parti. 
Le parti ouvrier de Québec fit ses débuts, à l'occasion d'une élection fédérale, dans le comté de Sainte-Marie de Montréal. Le candidat y fut défait, mais en 1906, le parti réussit à faire élire son premier (et son dernier) député fédéral, dans la personne d'Alphonse Verville, chef syndical de la section 144 de l'union des plombiers et président du Congrès des métiers et du travail. Des divisions internes, au cours de la Grande Guerre, et immédiatement après, ont amené la disparition du parti au début des années 1920. Le vide laissé par sa mort n'a jamais été comblé.

L'agitation que le Québec a connue autour de l'imposition de la conscription en 1917 est un fait bien connu. Il faut souligner que le mouvement anti-conscriptionniste n'a jamais obtenu un appui solide dans le mouvement ouvrier, même si le Conseil des métiers et du travail de Montréal a exprimé sa violente opposition à la conscription et participé à l'organisation d'au moins une démonstration avant que la loi ne fut adoptée, à la fin du mois d'août 1917.

La position équivoque adoptée par le Congrès des métiers et du travail, au niveau canadien, l'option très favorable à la guerre et à la conscription de Gompers et des chefs de la Fédération américaine du travail aux EtatsUnis, ont contribué à ralentir la campagne anti-conscriptionniste des succursales locales du Québec et des membres québécois des unions internationales. Le Conseil des métiers et du travail de Montréal accorda son appui à la grève générale de Winnipeg, malgré la froideur, l'opposition même, de certains chefs syndicaux montréalais. R.J. Johns, président du conseil de Winnipeg, avait passé plusieurs semaines à Montréal, et un grand ralliement avait été organisé par le Conseil de Montréal au parc Jeanne-Mance, en juillet 1919. Les orateurs invités étaient deux organisateurs de la grève de Winnipeg, A.A. Heaps et R.E. Bray. Après la fin de la grève, un montant de $100 \$$ fut donné pour aider les familles des grévistes encore en prison. L'appui apporté fut bien au-dessous de ce qu'avaient espéré les chefs de Winnipeg et de ce à quoi on aurait pu s'attendre, à l'occasion d'un tel conflit historique. La montée des travailleurs canadiens, surtout dans l'Ouest, et le militantisme qu'on y a remarqué, la dernière année de la guerre et les années qui l'ont suivie, n'ont jamais rejoint le mouvement anticonscriptionniste du Canada français et n'ont jamais réalisé d'alliance avec lui.

\section{DE LA FIN DE LA PREMIÈRE GRANDE GUERRE À LA FIN DE LA DEUXIÈME}

Hollywood a qualifié les années ' 20 de décennie ronflante («Roaring Twenties»). Pour les travailleurs et le mouvement syndical du Québec, le mot de Jamieson, les années engourdies («Torpid Twenties»), semble beaucoup plus approprié. Depuis la grave récession de 1921-1922 jusqu'au début de la grande dépression de 1930, la décennie de 1920 se résume, pour les travailleurs, en une succession de baisses de salaire et de périodes de chômage, en un mot en une lutte sans fin pour joindre les deux bouts. Pourtant, les revenus des entreprises et des investisseurs prospéraient à qui mieux mieux. 


\section{L'expansion industrielle des années '20}

Ce fut une période de grande expansion industrielle. De nouveaux centres urbains jaillirent presque du jour au lendemain. Des villes de compagnie surgirent au milieu de la campagne, comme à Noranda, Arvida, Baie-Comeau, Drummondville et Riverbend (aujourd'hui partie d'Alma), à cause du cuivre, de l'aluminium, de la pulpe et du papier, des textiles synthétiques. Tous ces investissements reposaient d'abord sur du capital et du management américain, mais aussi sur quelques entreprises dont le siège social était à Toronto, comme celles qui ouvrirent les mines de métaux du nord-ouest du Québec. En fait, si l'on excepte la basse Côte-Nord et la région de Chibougamau, dont le développement n'a commencé qu'après la seconde guerre mondiale, aucune partie du Québec, aujourd'hui industrialisée, n'avait été intouchée, durant les années '20, par la pénétration du capital américain.

L'attrait principal pour les investisseurs, c'était la présence de ressources naturelles considérables, à bon prix et d'accès facile. Ces ressources incluaient les pouvoirs hydro-électriques pour l'aluminium et l'industrie chimique, ainsi que les forêts pour l'industrie du papier et des textiles synthétiques. Une autre source d'attrait résidait dans la main-d'oeuvre à bon marché que fournissaient les mères prolifiques du Québec, dans leur docilité aux enseignements de l'Église. Le gouvernement canadien se mit en frais, au début des années 1920, de vendre aux investisseurs potentiels des États-Unis cette importante ressource, par exemple dans le texte suivant:

Nulle part ailleurs qu'au Québec peut-on trouver des conditions de travail plus stables et plus satisfaisantes du point de vue des manufacturiers. La main-d'oeuvre est abondante... les canadiens-français sont une race industrieuse et économe, et l'enseignement religieux les éloigne de toute association nuisible avec les organisations internationales.

L'expansion industrielle des années '20 créa tout près de 25000 emplois additionnels dans le secteur manufacturier, soit une augmentation de $12 \%$. Le gros de l'expansion se produisit dans la dernière partie de la décennie; la récession désastreuse de 1921-1922 avait, pour sa part, fait perdre 40000 emplois dans les manufactures, soit une diminution de près de $25 \%$.

\section{L'impact sur la classe ouvrière}

L'expansion industrielle en province, dans des régions jusqu'alors intouchées par la grande industrie, a provoqué un nouvel exode de la population des fermes et des villages vers les nouvelles manufactures. Ces groupes constituaient une addition importante à la classe ouvrière. Contrairement à la situation à Montréal, ces nouveaux travailleurs industriels étaient presque tous canadiens-français; pourtant, la haute direction et bon nombre de surintendants demeuraient anglophones, plusieurs venant d'en dehors de la province. C'était le cas également pour la très grande majorité du personnel professionnel et technique. À la fin de la décennie, les canadiens-français demeuraient minoritaires parmi les ingénieurs, et ils étaient sous-représentés dans les postes d'employés de bureau et dans les 
principaux métiers qualifiés, comme les outilleurs, les ajusteurs, les modeleurs, les inspecteurs de métaux, les chauffeurs de bouilloire ou ingénieurs stationnaires et les graveurs.

Il n'y avait là rien de surprenant, compte tenu du système de stratification selon la langue et du manque de développement dans le régime public d'éducation pour les enfants francophones. En 1926, alors que l'Ontario dépensait $5.29 \$$ par personne en frais d'éducation, de santé et de bien-être social, le Québec n'y accordait qu'exactement 1.00\$

Le manque à gagner ou déficit chronique qu'on pouvait observer entre les gains annuels (compte tenu du travail à temps partiel et du chômage) et le coût d'un budget familial normal se poursuivit durant toute la décennie de 1920. Lors de la récession de 1921-22, les gains moyens d'un chef de famille de la classe ouvrière à Montréal allaient de $960 \$$ par homme de métier dans le bâtiment à $1565 \$$ pour un surintendant de premier niveau. Le budget d'une famille moyenne était estimé à $1600 \$$. Dans les années de prospérité de 1928-1929, avec un taux de chômage relativement faible, les deux tiers des ouvriers adultes de Montréal, travaillant à plein temps, gagnaient moins de 1300 \$, ce qui était de beaucoup inférieur aux 1590 \$ jugés nécessaires pour les besoins d'une famille moyenne.

Le profil qu'a tracé Terry Copp de la classe ouvrière industrielle de Montréal - profil qui pourrait s'appliquer aussi bien aux autres centres urbains de Québec - est particulièrement révélateur: une culture de pauvreté, créée à la fois par les revenus qui ne dépassaient pas le niveau de subsistance et par l'absence de sécurité d'emploi.

\section{Le mouvement syndical des années '20}

Si on les compare aux périodes précédentes d'expansion, les années '20 auraient da être témoins d'un fort niveau d'organisation syndicale et de militantisme. En fait, c'est le contraire qui s'est produit au Québec, tout comme dans le reste du Canada et aux Etats-Unis. La tendance générale a été celle d'une chute dans les trois mesures suivantes, la représentation (le nombre de membres par rapport à la main-d'oeuvre), le militantisme et l'efficacité.

Si l'on excepte la Fraternité canadienne des employés de chemins de fer, le mouvement syndical au Québec était principalement constitué d'organisations affiliées à la Fédération Américaine du travail (FAT) et au Congrès des Métiers et du Travail du Canada (CMTC). Les autres unions dites nationales et la CTCC n'avaient guere plus qu'une importance marginale. On peut se demander pourquoi les unions internationales bien établies (à l'exception de celles de l'industrie du vêtement) ont manifesté si peu d'intérêt et investi si peu de ressources pour organiser les travailleurs non syndiqués du Québec.

Il y avait des circonstances objectives qui défavorisaient l'organisation syndicale à grande échelle. L'idéologie dominante de la libre entreprise, avec l'appui du gouvernement et des médias d'information, contribuait à créer un climat hostile aux syndicats et aux unions. De plus, l'expansion se 
faisait principalement dans des industries jeunes et dynamiques à production de masse, caractérisées par une forte concentration du capital et une division du travail très poussée. La grande majorité des ouvriers dans ces géants nouveaux - l'automobile, l'équipement électrique, le caoutchouc, le verre, les produits chimiques, la transformation des aliments, l'aluminium et le cuivre - tout autant que dans les anciennes industries, comme dans l'acier, étaient principalement semi-qualifiés ou non qualifiés. Les employeurs y étaient fortement opposés à la syndicalisation et ils avaient la possibilité de traduire cette opposition dans la réalité.

Il y avait aussi des facteurs subjectifs. Pour organiser ces travailleurs, les unions en place devaient avoir non seulement une motivation profonde, mais elles devaient aussi effectuer un changement radical dans leurs structures et leur orientation. La nouvelle classe ouvrière ne trouvait vraiment pas sa place dans la formule des vieilles unions de métiers, dont l'objectif était de conserver le plus grand nombre possible d'emplois pour leurs membres qualifiés, ce qu'elles réalisaient par des lignes de démarcation très sévères entre les métiers, calquées sur le modèle des corporations d'autrefois. L'existence d'une telle situation était particulièrement évidente aux États-Unis dans les années qui ont précédé immédiatement la création du Congrès des organisations industrielles (CIO). En fait, c'est cette situation même qui a amené la naissance et la croissance extraordinaire de cette nouvelle centrale.

Au Québec, comme si c'était nécessaire, il y avait une autre désincitation, la question de la langue. Les représentants des sections locales demandaient constamment des représentants syndicaux pouvant parler français. Ceci aurait exigé des dépenses additionnelles et entraîné une sorte de statut spécial pour les unions du Québec, autant de péchés graves pour les dirigeants de la Fédération américaine du travail.

La décennie de 1920 et les premières années 1930 ont vu se développer bon nombre de syndicats de boutique, suite à une nouvelle tactique des employeurs en vue de garder leurs établissements ouverts, sans la présence d'aucune union. L'ironie du sort, c'est que le procédé a entraîné un effet de boomerang, alors que plusieurs de ces syndicats de boutique, ayant déjà la forme d'un syndicat industriel ouvert à l'ensemble des salariés, sont passés au mouvement syndical de bonne foi. Au Québec et en Ontario, plusieurs sections locales des ouvriers de l'acier ont été formées par l'affiliation d'anciens syndicats de boutique aux Métallurgistes unis d'Amérique. Sous cet aspect, les syndicats catholiques ont profité de leur structure, qui était de type industriel; dès le début, ils avaient opté pour ce type de structure, parce qu'il convenait mieux à un mouvement idéologique, qui cherchait à rejoindre tous les travailleurs de chaque établissement, sans égard a leurs occupations respectives.

Il n'y a donc rien d'étonnant à constater que les quelques grèves importantes des années 1920 se sont produites dans les secteurs traditionnels du vêtement et de la chaussure. Dans la ville de Québec, le premier syndicat catholique, créé en 1901 par le Cardinal Bégin, disparut à la suite d'une grève amere, commencée et poursuivie contre la volonté des directeurs du mouvement et de l'Eglise. L'industrie devait demeurer sans organisation 
syndicale durant les 25 années suivantes. À Montréal, les Travailleurs amalgamés du vêtement, un groupe très militant, menèrent 5000 ouvriers à travers une série de grèves échelonnées sur une période de 10 mois en 1926-1927.

\section{La grande dépression}

La grande dépression dura 10 ans. Ces années terribles, pratiquement toutes les familles ouvrières du Québec en ont subi le choc dévastateur. Au plus creux de la vague, le taux de chômage à Montréal était d'au moins $25 \%$; dans le quartier ouvrier de Saint-Henri, juste en bas de la côte où loge Westmount, il y en avait près de $50 \%$ en chômage. Plus important encore est le fait que la très grande majorité des ouvriers furent en chômage à un moment ou l'autre, et souvent pour de longues périodes, alors qu'un nombre indéterminé le fut littéralement pendant plusieurs années. Ceux qui ont vécu cette catastrophe ne peuvent pas oublier cette forme d'attaque, qui grandissait avec le temps, à la vie humaine, à la santé et à la dignité des travailleurs.

Il n'y avait pas alors d'assurance-chômage. Au début de la dépression, le premier ministre King avait déclaré, en avril 1930, que son gouvernement ne dépenserait pas $0.05 \mathfrak{C}$ pour ceux qu'on appelait les chômeurs. Aux organisations charitables de s'occuper du problème. L'oeuvre de la soupe, organisée par différentes sociétés catholiques de bienfaisance, devait en nourrir des milliers chaque semaine. Quand ces efforts eux-mêmes s'écroulèrent, les municipalités durent assumer le fardeau de fournir le secours direct. En octobre 1934, 38000 familles de Montréal firent application pour ce genre de secours. Les tempêtes de neige étaient accueillies comme des événements providentiels, parce qu'elles fournissaient un peu de travail aux chômeurs: les quelques privilégiés qui pouvaient ainsi se trouver du travail recevaient $0.50 \$$ du voyage, mais ils devaient fournir eux-mêmes la voiture et le cheval.

Entre 1930 et 1933, les taux de salaires diminuèrent d'environ $15 \%$, alors que l'indice du coût de la vie tomba de $22 \%$. Ces chiffres signifient peu de chose, puisqu'ils ne reflètent pas l'effet du chômage prolongé et des courtes périodes de travail. De toute manière, les taux de salaires mentionnés ne correspondaient pas nécessairement aux salaires effectivement payés. Même dans les métiers syndiqués, plusieurs travailleurs étaient obligés d'en remettre une partie à leur employeur.

Les unions en place, déjà en déclin avant la dépression, se sont pratiquement désintégrées sous l'effet de la crise. Des groupes aussi puissants que l'Union des mineurs, les Travailleurs amalgamés du vêtement, et bien d'autres, n'avaient pratiquement plus de membres en 1933.

Faute de résistance efficace à la situation de la part des unions et des syndicats en place, la Ligue pour l'unité des travailleurs du parti communiste a eu, de 1930 à 1934, un quasi-monopole sur l'activité de grève durant les pires années de la grande dépression. Même au Québec, qui n'était certainement pas au coeur des activités de la Ligue, il y eut quelques grèves importantes en 1934, comme la grève des bûcherons, la grève contre 
la mine Noranda, et le débrayage de milliers d'ouvriers de l'industrie du vêtement pour dames, à Montréal. La Ligue eut une vie courte mais courageuse, avant qu'elle ne soit dissoute et ses différentes unités intégrées dans des unions existantes; la Ligue n'a pas produit de résultats immédiats d'importance. Cependant, elle a développé, entraîné et animé un nombre étonnant de militants de la base et d'organisateurs professionnels. Ils ont joué un rôle important dans la reprise et le renouveau du mouvement syndical, spécialement dans le CIO, jusqu'au moment des purges anticommunistes de 1949-1952, au sommet de la guerre froide entre les grandes puissances. D'autres organisations du parti communiste du Canada pour les chômeurs ont également produit de futurs chefs syndicaux pour les années à venir.

La diminution dramatique des unions internationales au Québec eut pour effet, entre autres, d'accroître l'influence relative de la CTCC. En 1934, sous la pression de la CTCC et de certains ecclésiastiques, le gouvernement du Québec adopta la Loi de la convention collective, unique en Amérique du Nord, à ce moment et encore aujourd'hui. Cette loi visait à établir un certain degré d'uniformité dans les salaires et les heures de travail, en se basant sur certaines conventions collectives conclues dans des industries à forte concurrence comme la construction, l'imprimerie et le vêtement; ces industries avaient déjà une assez longue histoire de conditions de travail déterminées par la négociation syndicale. Au début des années 1930, ces industries vivaient en quelque sorte la loi de la jungle, et très peu d'employeurs pouvaient concurrencer les ateliers dits ouverts. Les vieilles unions de métiers abhorraient ce genre de loi, à cause de son inspiration corporatiste et de leur propre philosophie de base fondée sur la méthode bilatérale. Quoi qu'il en soit, même ces unions ont dû avoir recours à cette loi pour donner un minimum de protection aux quelques membres qu'il leur restait et aux employeurs qui demeuraient syndiqués.

\section{La seconde moitié des années '30}

Le point tournant fut 1935 . Les débuts d'une reprise hésitante, les efforts de l'administration Roosevelt avec le «New Deal" et son "Wagner Act» (Loi nationale sur les relations ouvrières), forçant les employeurs à reconnaître un agent négociateur accrédité et à négocier avec lui une convention collective, tout cela fournissait le contexte nécessaire à l'étincelle qui allait produire une véritable explosion dans l'organisation et le militantisme syndical. Les trois lettres CIO devinrent le mot de passe, en français et en anglais; elles apportaient aux travailleurs québécois la nouvelle que des millions d'ouvriers dans les industries de production de masse entraient dans les rangs des unions industrielles, sortaient en grève ou $s^{2}$ installaient dans leurs usines dans de mémorables grèves-sur-le-tas.

Au Québec comme en Ontario, l'absence de loi correspondant au "Wagner Act» américain, tout autant que le climat politique, empêchèrent l'exemple des États-Unis d'être suivi, du moins immédiatement. L'organisation syndicale se heurtait toujours à la résistance patronale, comme auparavant, et il fallait encore recourir à la grève pour se faire reconnaître, comme il fallait toujours faire face à l'intervention inévitable de la police et de l'ap- 
pareil judiciaire. Résultat: les percées dans les grandes industries se limitaient aux secteurs où le syndicalisme était déjà établi avant la dépression. Même là, il fallait souvent recourir à la grève. Tel fut le cas dans l'industrie du vêtement pour dames à Montréal, en 1937. Une grève de 5000 ouvriers et ouvrières força l'association patronale à se rendre. Finalement, une entente durable fut obtenue suite à une seconde grève en 1940.

Les développements les plus spectaculaires, dans la seconde moitié des années 1930, se produisirent en dehors de Montréal; ils impliquaient les syndicats catholiques, qu'on considérait toujours comme opposés à la grève et favorables à l'harmonie entre les classes. Des syndicats de la CTCC s'étaient établis dans les grandes fabriques de coton, dans les mines d'amiante, dans les raffineries d'aluminium de la région du Saguenay, ainsi que dans les chantiers maritimes et les aciéries de la famille Simard a Sorel. Bien que plusieurs grèves fussent du type grève sauvage et spontanée, la grève de 1937, qui impliquait 10000 travailleurs de la Dominion Textile, à travers toute la province, était autorisée et supportée par le clergé catholique, au moyen de collectes faites dans les églises après les messes du dimanche. Â la fin, le Cardinal Villeneuve intervint lui-même et persuada le premier ministre Duplessis et la compagnie Dominion Textile d'accepter un règlement. Le règlement mit fin à la grève, et au syndicat. Le désappointement et le ressentiment des grévistes allaient éloigner la CTCC des fabriques de coton pour de nombreuses années.

L'orientation première de la CTCC vers l'harmonie entre les classes ne disparut pas du jour au lendemain. Il y eut un certain nombre de conflits amers avec les unions internationales, dans lesquelles des employeurs utilisèrent des syndicats affiliés à la CTCC ou conclurent avec eux des accords dits de compérage («sweetheart deals»). Cependant, la transformation qui avait commencé à s'effectuer dans les années qui précédèrent immédiatement la guerre s'avéra une transformation réelle et profonde, qui se renforça et s'étendit dans la période d'après-guerre.

La conclusion à tirer c'est que le mouvement syndical catholique au Québec a adopté, comme il l'a fait en France, en Italie et ailleurs, le mode de comportement des organisations ouvrières dominantes, afin d'y survivre et d'y jouer un rôle. C'était tout particulièrement le cas en Amérique du Nord où les employeurs ne voulaient pas de véritables unions ou syndicats, alors que, naturellement, les travailleurs voulaient que leurs syndicats et leurs unions soient de véritables syndicats et de véritables unions.

Les nouvelles unions affiliées au CIO s'implantèrent au Québec avec la deuxième guerre mondiale. En conséquence, la rivalité amère qui, en 1938, existait aux États-Unis entre la Fédération américaine du travail et le Congrès des organisations industrielles - rivalité qui conduisit, en 1939, à l'expulsion du CMTC des succursales locales canadiennes affiliées au CIO, suite à un ultimatum de la FAT - eut relativement peu d'impact au Québec. D'un autre côté, l'établissement du Congrès canadien du travail (CCT) en 1940, par les sections canadiennes des unions affiliées au CIO et par les unions canadiennes du Congrès pan-canadien du travail devait fournir une base solide à la fois pour organiser les non-syndiqués et pour alimenter les rivalités entre, d'une part, le CMTC et le CCT, et d'autre part, la CTCC et CCT. 
Le climat politique a changé d'une façon dramatique dans les années qui ont immédiatement précédé la guerre. Le gouvernement libéral de Louis-Alexandre Taschereau fut défait en 1935 par une étrange coalition. Le couple insolite se composait du parti conservateur du Québec, dirigé par Maurice Duplessis, et d'un groupe de jeunes turcs, intellectuels et anciens libéraux qui avaient abandonné le régime corrompu de Taschereau. Le programme de cette nouvelle coalition, connue sous le nom d'Union nationale, comportait la nationalisation des compagnies de pouvoirs électriques et un certain nombre d'autres mesures à caractère nationaliste et populiste. Une fois au pouvoir, Duplessis écarta simultanément les promesses radicales qu'il avait pu faire et les hommes qui les avaient inspirées; il s'installa au pouvoir en y créant un nouveau régime, encore plus conservateur, antisyndical, et éventuellement corrompu que celui de son prédécesseur. Toutefois, Duplessis, en fin renard qu'il était, conserva une partie de son ancienne rhétorique, autonomiste et nationaliste (entendez anti-Ottawa), avec des relents populistes. La rhétorique ne l'empêcha pas d'inviter les capitalistes américains et autres capitalistes du monde à venir festoyer, grâce aux générosités du Québec à leur endroit en matière de ressources naturelles, $d$ 'ententes fiscales et autres privilèges financiers, sans exclure les services de la police provinciale et de l'appareil gouvernemental contre les unions et les grèves. Duplessis développa également, à un degré rare, l'anticommunisme et la peur de tout ce qui était rouge; il s'en servait en toute occasion, allant jusqu'à accuser Jean Marchand d'être lui-même un rouge.

\section{Les années de guerre (1939-1945)}

L'organisation syndicale à grande échelle dans les industries de production de masse commença vraiment au Québec autour de 1940. La guerre avait absorbé la plupart des chômeurs, et des milliers de nouveaux emplois s'ouvraient dans les usines; plusieurs de ces emplois étaient accessibles même aux femmes. Une autre vague d'immigrants de la campagne s'engouffra à Montréal et dans les autres centres urbains, où se faisait l'embauchage pour ces nouvelles industries. Des établissements géants, employant 10000 personnes ou plus, surgirent presque du soir au matin. Comme les établissements de guerre devaient normalement disparaître avec la fin de la guerre, et qu'on s'attendait au contrôle des salaires sous peu, la direction de ces entreprises ne combattait pas l'organisation syndicale au même degré qu'elle avait pu le faire dans les industries de paix. L'arrêté-enconseil CP 1003, inspiré du "Wagner Act» américain, ne fut adopté qu'en 1944, mais il existait déjà, avant cette adoption, une certaine pression de la part du gouvernement sur la direction des entreprises en vue de les amener à reconnaître les unions et à négocier avec elles.

$\mathrm{Au}$ Québec, la faiblesse des syndicats affiliés à la CTCC à Montréal et la jeunesse des succursales du CIO, affiliées au Congrès canadien du travail, donnaient aux unions du CMTC un avantage marqué; il leur suffisait de prendre la peine d'en profiter et, tout particulièrement, d'opter pour les structures et les méthodes syndicales de type industriel. C'est ce qui se produisit. Un organisateur de talent, Robert Haddow, membre de l'Association internationale des machinistes et militant de longue date dans le parti 
communiste du Canada, avait acquis une solide connaissance des stratégies et des tactiques syndicales dans l'Écosse de son enfance; il réussit à persuader l'Association des machinistes de lancer une grande campagne d'organisation, principalement mais uniquement, dans la région de Montréal. Dans un temps relativement court, 50000 travailleurs avaient signé leur carte de membre de l'union. Un certain nombre d'organisateurs volontaires, membres du parti communiste ou des organisations de jeunes communistes, avaient envahi les établissements industriels pour y être embauchés et y faire de l'organisation syndicale. Les établissements qui comptaient quelques milliers de travailleurs furent ainsi organisés de l'intérieur. La campagne s'étendit des industries de guerre aux industries de paix. On organisa les travailleurs du textile, des salaisons, des industries chimiques, du tabac et des appareils électriques. Les unions de la pulpe et du papier connurent un regain de vie: elles réapparurent dans la plupart des moulins importants.

À la fin de la guerre, les syndiqués au Québec étaient quatre fois plus nombreux qu'avant la grande dépression. Plus important, des unions et des syndicats existaient maintenant dans tous les coins et recoins de la province, de même que dans la plupart des grandes industries de base. Dans le secteur de l'administration publique, les employés des municipalités et des entreprises de transport urbain étaient solidement organisés à Montréal et dans quelques autres villes.

Les communistes et leurs alliés occupaient des positions importantes dans plusieurs unions affiliées au CMTC; ils constituaient une opposition sérieuse au Conseil de Montréal - rappelons que la Fédération provinciale n'avait à ce moment qu'un pouvoir marginal - et ils exerçaient une influence réelle au sein du CMTC lui-même. Cependant, leur position était plus fragile qu'il ne paraissait. Leur force était concentrée dans quelques unions; ils étaient absents des unions d'employés de chemins de fer, des métiers de l'imprimerie. Ils étaient absents ou faibles dans la grande majorité des sections locales des métiers du bâtiment. Au fur et à mesure que les établissements de guerre fermaient leurs portes, les grosses succursales locales que Haddow avait établies s'évanouissaient. Quand arriva le moment critique, peu de temps après la fin de la guerre, le rapport de force s'avéra inégal. L'artillerie lourde des sièges sociaux des unions internationales entra en action, pleinement supportée par le gouvernement Duplessis et tout son appareil. Les purges à l'intérieur même des unions et la condamnation par le gouvernement des unions soupçonnées d'être dirigées par des communistes réussirent à isoler ceux-ci et à les éliminer, de même qu'à annuler leur influence dans les deux groupes du mouvement syndical non catholique, c'est-à-dire dans les unions du CMTC et dans celles du CCT.

\section{DE LA FIN DE LA DEUXIÈME GUERRE MONDIALE Ả CE JOUR}

Les transformations les plus importantes et les plus spectaculaires du mouvement syndical au Québec se sont produites dans les deux décennies de 1950 et de 1960 . Les développements depuis 1971 n'ont fait que renforcer et 
accentuer les tendances déjà engagées. Les historiens de l'avenir reconnaîtront peut-être que l'année 1983 aura marqué un autre tournant, mais il est trop tôt pour l'affirmer'.

\section{Le régime Duplessis (1946-1959)}

La CTCC continua dans la nouvelle voie qui s'était manifestée au milieu des années '30. En 1946, le groupe de ceux qui favorisaient une rupture avec le passé assuma la direction de la centrale. Avec Gérard Picard comme président et le jeune Jean Marchand comme secrétaire, la Confédération se donna, pour la première fois de son histoire, des services techniques appropriés, en embauchant une équipe de jeunes canadiensfrançais, formés à l'université, dont les idées penchaient à gauche. La CTCC elle-même fit l'objet d'une restructuration importante: elle devint la centrale syndicale la plus centralisée au Québec, si ce n'est même au Canada. La célèbre grève d'Asbestos, en 1949, a consommé la rupture entre la CTCC et Duplessis. Pendant les 10 années qui suivirent, la CTCC, pourtant anti-communiste, devint l'adversaire principal de Duplessis et sa cible favorite. Des grèves nombreuses et amères, en plus du harcèlement de la part du gouvernement, ne manquèrent pas de faire sentir leurs effets.

En 1956, quand le CMTC et le CCT se regroupèrent pour former le nouveau Congrès du travail du Canada (CTC), la CTCC était encore bien vivante et faisait toujours parler d'elle, mais guère plus. Le nombre de ses membres était demeuré stationnaire depuis quelques années, ce qui représente toujours un sérieux danger pour une organisation syndicale dynamique. À son congrès de 1956, les délégués votèrent en faveur de l'unité organique du mouvement syndical canadien. Cette unité devait se réaliser par l'affiliation en bloc de la CTCC au CTC, avec la garantie du statut d'union nationale au sein du Congrès, comme la Fraternité canadienne des employés de chemins de fer. Toutefois, plusieurs années de négociations infructueuses ont montré qu'il y avait beaucoup de résistance à ce projet, tant chez les unions internationales qu'à l'intérieur même de la CTCC. Au moment crucial, la Providence prit les choses en main. Duplessis mourut subitement le jour de la Fête du travail de 1959. La fin d'une ère s'amorçait; elle fut précipitée par la mort également subite de son successeur Paul Sauvé, après un régime de 100 jours d'une sorte de «New Deal» québécois.

En juin 1960, le parti libéral de Jean Lesage prit le pouvoir. Ce fut le début de la révolution tranquille. La grande majorité du mouvement ouvrier lui accorda un appui enthousiaste, particulièrement la CTCC sous l'influence de Jean Marchand. Le nouveau climat politique favorisait le monde du travail. La nouvelle CSN (l'ancienne CTCC) tirait profit de la situation, mais toutes les unions appréciaient le nouveau courant d'air frais. Quelques-uns se laissèrent emporter dans leur approbation sans réserve du régime, surtout depuis que René Lévesque, qui passait pour l'homme des syndicats, était entré au cabinet.

2 Le professeur Léo Roback a donné cette communication au début de 1983. À ce moment, le gouvernement du Québec venait d'adopter une série de lois contraignantes visant divers syndicats du secteur public. (Note du traducteur.) 
Pendant tout son règne, de 1944 à 1959, Duplessis avait utilisé la stratégie de «diviser pour régner», dans ses rapports avec les unions et les syndicats. À la fin de son règne, ses politiques et ses gestes déchaînés contre le mouvement syndical et contre la classe ouvrière, comme l'utilisation brutale des forces policières pour briser la grève des Métallurgistes unis d'Amérique, à la mine Noranda de Murdochville, avaient eu pour effet de rapprocher des organisations ouvrières rivales et en conflit. Ses propres supporteurs au sein des unions ne pouvaient plus parler et se trouvaient isolés. Une situation analogue semble se développer à l'heure actuelle chez les supporteurs du Parti québécois au sein des organismes syndicaux.

La croissance des organismes syndicaux entre 1946 et 1959 fut plus lente au Québec qu'en Ontario. Toutefois, pour paraphraser le docteur Johnson, la merveille c'est que les syndicats et les unions du Québec aient pu réaliser tout ce qu'ils ont réussi à faire, malgré les obstacles incroyables soulevés par les employeurs à leur endroit, en pleine collusion avec l'appareil gouvernemental. On pourrait écrire des volumes sur l'histoire de la Commission des relations ouvrières, à cette époque, où elle a accrédité des syndicats de boutique sans hésitation, refusé l'accréditation à des unions qui avaient une claire majorité, et désaccrédité d'autres unions avant même qu'une demande à cet effet ne soit déposée, sans entendre l'organisme concerné.

\section{Les années de la révolution tranquille}

En 1960, au début de la révolution tranquille, le nombre total de syndiqués s'élevait au Québec à environ 400000 . La CSN en avait 100000 , et les affiliés du CTC, 250000 . À la fin du régime de Jean Lesage, en 1966, il y avait, en tout, 520000 syndiqués, soit un accroissement de $25 \%$. Mais les syndicats CSN avaient doublé le nombre de leurs membres. Cela était dû largement à l'entrée de dizaine de milliers de travailleurs du secteur public, notamment dans la fonction publique et dans les hôpitaux. En 1972, avant la crise interne et le mouvement de sécession qu'a connu la CSN, le nombre de ses membres atteignait un sommet de 350000 , pour un total de 900000 syndiqués au Québec.

Entre temps, la Fédération des travailleurs du Québec, fondée en 1957, du mariage obligé des deux fédérations de l'ancien CMTC et de l'ancien CCT, sous l'oeil menaçant des deux organismes canadiens, grandissait en nombre et en influence. Sa longue lutte pour obtenir un statut spécial à l'intérieur du CTC, finalement réalisé en 1974, a cristallisé son importance grandissante et son autorité, alors que celle du CTC déclinait au Québec. À l'heure actuelle, les affiliés de la FTQ comptent environ 425000 membres, comparés aux 225000 de la CSN, du moins si l'on ne tient pas compte de l'effet des mises à pied et des fermetures d'usines récentes.

Le troisième groupe en importance dans le mouvement syndical québécois est formé de la Centrale de l'enseignement du Québec (CEQ). De 1946 à 1971, cet organisme s'est modifié profondément: au début, c'était une fédération aux liens assez lâches d'associations locales de professeurs catholiques; elle a évolué pour devenir une véritable centrale syndicale, 
hautement structurée et très bien équipée; certains la considèrent comme l'aile gauche du mouvement ouvrier du Québec. Au début des années 1970, les membres de la CEQ étaient les plus importants supporteurs du Parti québécois; mais la CEQ est devenue l'ennemi public numéro $1 \mathrm{du} P Q$.

De façon assez paradoxale, le programme de la révolution tranquille, qui voulait introduire des réformes de toute urgence pour redonner au Québec un statut de société industrielle moderne, correspondait assez bien aux demandes que le mouvement ouvrier avait formulées à grands cris tout au long du régime Duplessis. En fait, la révolution tranquille a volé en quelque sorte le programme du mouvement ouvrier et a laissé celui-ci bouche bée. En 1965, un an avant sa défaite aux élections, le gouvernement LesageLévesque montrait des signes de fatigue et d'impasses. Il reculait de plus en plus souvent sur différentes questions et les affrontements avec le mouvement ouvrier se multipliaient.

\section{Le PQ ou un parti ouvrier?}

Cette période d'hésitations et de doutes a coïncidé avec la naissance de différents partis et mouvements indépendantistes. Quand René Lévesque fonda ce qui devait devenir le Parti québécois, suite à une fusion de différents groupes, de droite et de gauche, il rejoignait un vaste auditoire d'intellectuels, de membres et de représentants syndicaux, tout particulièrement dans les rangs des Métallos, de la CEQ et de la CSN. La popularité personnelle de René Lévesque auprès des ouvriers et des représentants syndicaux a joué un rôle décisif pour cristalliser les courants et les sentiments nationalistes de larges couches de la classe ouvrière.

Quelques années plus tôt, dans les milieux syndicaux du Québec, un début d'enthousiasme pour le Nouveau Parti démocratique (NPD) s'était manifesté lors de sa fondation, en 1961; mais il se refroidit rapidement, au fur et à mesure que le débat sur la question nationale s'échauffait. Le Québec était-il une des dix provinces canadiennes, ou une des deux nations fondatrices? Le congrès de Québec, en 1963, devait établir une section du NPD-Québec, avec l'appui particulier du mouvement syndical; le projet échoua. Le congrès s'empêtra dans les implications pratiques des problèmes de structures du NPD-Québec et, par-dessus tout, dans la question de son autonomie par rapport au parti fédéral. Le NPD n'a jamais pris son envol au Québec. La place qu'il devait occuper a bientôt été remplie par le Parti québécois (PQ). Le programme social du $\mathrm{PQ}$ et sa structure démocratique avaient des ressemblances avec le NPD; mais le PQ n'avait aucun rapport organique avec la classe ouvrière organisée, et celle-ci n'avait aucun input direct dans le parti.

En 1970, une série de conférences régionales sur l'action politique fut organisée conjointement par les trois centrales. La série devait connaître son point culminant dans une réunion à Montréal, à l'automne de cette annéelà. On parlait même et on espérait, en certains milieux, que ce serait la rampe de lancement d'un parti ouvrier québécois. La conférence n'a jamais eu lieu, à cause de la crise d'octobre 1970. Toutefois, la position commune des trois organismes syndicaux contre la proclamation de la Loi des mesures 
de guerre et leurs réactions à une récession qui commençait, avec ses inévitables mises à pied, provoqua un développement remarquable. Chaque organisme syndical, à tour de rôle, à quelques mois d'intervalle, publia un manifeste. Ces trois documents présentent des analyses critiques du système actuel et formulent des propositions pour une transformation radicale de la société québécoise, dans le sens d'un Québec socialiste, d'un genre nouveau et original.

En résumé, les centrales syndicales ont cherché à combler le vide idéologique et politique causé par l'absence d'un authentique parti ouvrier; un tel parti constitue pourtant un élément essentiel du mouvement ouvrier. D'un autre côté, ce nouveau départ ne rallia pas tous les secteurs du mouvement syndical. Les groupes qui ont quitté les rangs de la CSN, avec près de la moitié de ses membres, en 1972 et 1973 - dont un a formé une nouvelle fédération (la Centrale des syndicats démocratiques, CSD) - se sont justifiés en faisant appel à la nouvelle tendance de la CSN, qu'ils qualifièrent de gauchiste et de politique. Il faut reconnaître aussi que ni la CSN ni la CEQ n'ont réussi à développer un appui substantiel dans l'ensemble de leurs membres en faveur d'un nouveau parti ouvrier. En conséquence, le Québec souffre présentement d'une sorte de vacuum politique.

La confrontation entre le Front commun des différents syndicats d'employés du secteur public (FTQ-CSN-CEQ) et le gouvernement du Parti québécois, qui a débuté à l'été 1982, a provoqué à la fois une défaite majeure pour les syndicats en cause et une sérieuse perte de popularité et d'appui pour le Parti québécois, y compris une baisse de près de $50 \%$ dans le nombre de ses membres. Les lois spéciales adoptées par l'Assemblée nationale incluaient des mesures qui prolongeaient unilatéralement les conventions collectives, imposaient des réductions de salaires allant jusqu'à $20 \%$ pour trois mois, et décrétaient de nouvelles conditions de travail, à la place et au lieu du renouvellement de contrats négociés. Le dernier coup, au début de 1983, fut l'adoption du Projet de loi 111. Cette mesure prévoyait, entre autre choses, la réduction de l'ancienneté pour les professeurs en grève, au rythme de trois ans pour chaque jour d'absence non autorisée, et une présomption de culpabilité. Maurice Duplessis a dû rougir dans sa tombe des mesures autrement modérées qu'il avait lui-même prises dans certaines circonstances de son temps. Aujourd'hui, en dépit des décisions judiciaires qui invalident certaines éléments de ces lois, des centaines de causes demeurent pendantes contre des professeurs individuels et leurs syndicats.

Il faut concéder que René Lévesque a réussi dans sa tactique de diviser pour régner, grâce à quelques erreurs de calcul de la part des dirigeants du Front commun. Une campagne de propagande, conteuse et très sophistiquée, visait à retourner les travailleurs du secteur privé contre leurs confrères et leurs consoeurs bien dodus du secteur public; à court terme, la campagne a donné d'importants résultats. Â la CSN, et tout particulièrement à la FTQ, les syndicats du secteur privé n'ont guère manifesté de sympathie envers les problèmes des professeurs syndiqués qui constituaient la cible principale du gouvernement. Sous cet aspect, le contraste entre le Québec et la Colombie-Britannique est frappant. 
La situation présente

Le gouvernement Lévesque, comme celui du régime Trudeau à Ottawa, cherche activement à rétablir un modus vivendi avec les syndicats, sous le thème général de la concertation, pour faciliter la reprise économique et la création d'emplois. La FTQ a répondu de façon positive, et elle reçoit encouragement et assistance financière pour son nouveau Fonds de Solidarité, un projet financé par les travailleurs pour fournir du capital aux entreprises en difficulté; la mesure vise à maintenir l'emploi. La CSN est profondément divisée sur la question de la concertation, alors que la CEQ est toujours réticente à s'embarquer.

Il est trop tôt pour dire à quel point et sous quelle forme la stratégie de concertation se développera. Également, on peut se demander si les appels à la souveraineté et au nationalisme, ainsi que la réapparition de Robert Bourassa à la tête du Parti libéral, suffiront pour vaincre ou au moins neutraliser le ressentiment, les désillusions et les désappointements qui se manifestent avec tant d'évidence dans les rangs du mouvement syndical envers le Parti québécois. Ce qui est clair, c'est que la lune de miel de 1976 est terminée, pour de bon, et qu'à sa place se sont installés le doute, la méfiance et l'hostilité. L'histoire généralement houleuse des travailleurs du Québec et de leurs divers mouvements semble sur le point de reprendre son cours habituel.

\author{
1986 NORTH AMERICAN LABOR HISTORY CONFERENCE \\ October 23 to 25,1986 \\ University of Toronto \\ Toronto, Canada
}

\begin{abstract}
1946 and After: North American Labor in the Post-War Years
Call for Papers
\end{abstract}

The eighth annual North American Labor History Conference will be held this year in Toronto, Canada on October 23-25, 1986. The conference title is "1946 and After: North American Labor in the Post-War Years". The conference's program committee invites proposals for papers. Please send a one page abstract. The deadline for submissions is April 1, 1986. The program will focus on five general themes: labor legislation, important strikes, women workers, labor and politics and the public sector. It is hoped these topics will encourage new work on post-war working class and labor history, some comparative (Canadian-American) papers and/or broader syntheses. Proposals should be sent to Professor Laurel Sefton MacDowell, History Department, Erindale College, University of Toronto in Mississauga, Mississauga, L5L 1C6, Canada. 